\title{
Stages of implementation of information modeling technology in the design organization
}

\author{
Petr Garyaev* \\ Moscow State University of Civil Engineering, Yaroslavskoe shosse, 26, Moscow, 129337, Russia
}

\begin{abstract}
The development of modern information technologies leads not only to an increase in the computing power of modern computers and the improvement of the capabilities of specialized software in the field of construction design, but also the need to develop approaches for the implementation of the proposed technologies at the stage of construction design.
\end{abstract}

\section{Introduction}

When developing a plan for a step-by-step plan for the implementation of information technology design, first of all, it is necessary to take into account the goals of implementing BIM in the organization, which will allow it to adequately assess the need to switch to design using BIM technology. Such a transition is justified if the customer needs an information model or the BIM format is specified in the design specification.

At present, many engineering firms have faced the fact that in the specifications in addition to the usual composition of design and working documents indicate the need for a BIM-model, so the transition to information modeling is becoming a critical aspect of ensuring competitiveness in the current market design and construction.

At the next stage, in addition to providing the design process with modern hardware and software, there is a need to understand how to create a digital model of a building, you need to know how to implement this new technology, taking into account the specifics of designing objects saturated with engineering equipment and systems. For example, in the case of designing curved designs in industrial and civil buildings, as a rule it expensive items, the cost of failure in the design of which is very high, three-dimensional the display BIM allows you to simulate different options coupling elements of designs with engineering systems. Visualization of three-dimensional model allows you to make these systems viable, that is, to design them in a way, to avoid possible collisions between structures and utilities in the construction and subsequent operation of the facilities.

An important task at the planning stage of BIM implementation is the need to implement joint work between sections of the project. This is one of the most difficult tasks in the implementation of BIM, since it is not easy to establish the exchange of data and combine them into a single whole, however, new technologies for converting and exchanging data make it possible to optimize this process and minimize the number of common design errors

${ }^{*}$ Corresponding author: garyaevpn@mgsu.ru 
- the same intersections of communication with the supporting frame and between itself, identifying and eliminating them at the design stage. In addition, it becomes possible to automate the calculation of the volume of materials. After the implementation of the model any object it is possible to calculate the volume area, the number of structures in the project and so on all the parameters, which may be necessary, which is an invaluable aid to the designer of the automatic compilation of estimates and statements.

Thus, the formation of the plan and the gradual introduction of information technology BIM design is becoming one of the main objectives of the project organization.

In the presented work, an attempt is made to form an approximate structure of a BIM implementation plan.

\section{Definition of scope and application plan for the step-by-step implementation of information technologies BIM design}

The proposed plan for the introduction of information technology the BIM design for project organizations and groups who gather in their practice to apply innovative design technology.

It should be noted that the proposed plan should certainly changeed, developed and supplemented on the basis of practice e of use.

The plan has the following goals:

- based on the analysis of many years of experience in the implementation of CAD and BIM technologies in construction, to propose the most effective plan for the implementation of BIM information technology in design practice;

- plan the training of specialists of various specialties participating in the implementation of BIM design, based on the formation of curricula and work programs for advanced training courses;

- increase the productivity of design work by creating standardized information modeling processes;

- to form an optimized information environment for BIM design based on various software, including highly specialized software;

- development of practical recommendations in order to ensure the uniformity of project information.

It should be noted that the implementation and implementation of the plan should be carried out by specialists with the necessary experience and qualifications.

\section{References to regulatory documents}

The development plan for the implementation of BIM should be based on the used AIs are following s standard s and guidelines governing information modeling:

- $\quad$ AEC (UK) BIM Protocol Project BIM Execution Plan, Version 2.0 September 2012;

- $\quad$ AEC (UK) BIM Technology Protocol, Version 2.1 June 2015;

- AIA Contract Document G202-2013 Building Information Modeling Protocol Form;

- BS 1192: 2007 Collaborative production of architectural, engineering and construction information. Code of practice;

- Employer's Information requirements. Core Content and Guidance Notes, Version 07 28.02.13, BIM Task Group;

- ISO / TS 12911: 2012 Framework for building information modeling (BIM) guidance;

- PAS 1192-2: 2013 Specification for information management for the capital / delivery phase of construction projects using building information modeling;

- $\quad$ Singapore BIM Guide, Version 2;

- $\quad$ The BIM Project Execution Planning Guide and Templates - Version 2.0, Pennstate; 
- $\quad$ THE PORT AUTHORITY OF NY\&NJ, Engineering Department, E / A Design Division BIM Standard, JUNE 2014;

- GOST R 1.4-2004 "Standardization in the Russian Federation. Organization standards. General Provisions ";

- GOST R 21.1101-2013 "System of design documentation for construction. Basic requirements for design and working documentation ".

- GOST R 10.0.03-2019 / ISO 29481-1: 2016 System of standards for information modeling of buildings and structures. Information modeling in the construction. Handbook for exchange of information. Part 1. Methodology and format

- GOST R 10.0.04-2019 / ISO 29481-2: 2012 System of standards for information modeling of buildings and structures. Building Information Modeling. Information Exchange Handbook. Part 2: Structure of Interaction

- SP 481.1325800.2020. A set of rules. Information modeling in the construction. Rules for application in cost- effective design documentation for reuse and when it is linked "( approved and put into effect by Order of the Ministry of Construction of Russia dated January 17,2020 N 18 / pr )

- GOST R 57310-2016 "Information modeling in construction. Delivery information guide. Methodology and format"

- GOST R 57311-2016 “ Information modeling in construction. Requirements for operational documentation of completed construction facilities "

\section{Basic terms and definitions}

- 2D - Object Model: Displays the geometry and position of objects on the plane (X and Y coordinates).

- 3D - a three-dimensional model of the object, created using the appropriate software products.

- 4D - model includes previous models, as well as a construction and installation process control system that allows you to control the construction process of an object, for example, the process of work in accordance with the network schedule, and also allows you to make operational changes to it if necessary.

- 5D - model - in addition to the 4D model, it allows you to manage the cost of construction and installation work, components and materials, thereby preventing unjustified overspending of financial and material resources during the construction of an object.

- 6D - concept for monitoring energy consumption throughout the construction and life cycle of a building to take optimal measures to reduce the building's energy consumption.

- 7D - ensures effective property management, including security monitoring, current and planned repairs and reconstruction.

- BIM- building information model: a digital representation of the physical and functional properties of a construction object, at all stages of its life - design, construction, operation, renovation and liquidation, serving as a source of collective knowledge for various specialists in the field of design, operation, modernization and etc..

- Building Information Modeling - the process of collectively creating and managing information about a building object, which forms the basis for decision-making throughout its life cycle.

- BIM project or information modeling project is a construction project developed using BIM technologies.

- Collision detection - the process of detecting design errors, according to standardized or other parameters, for example, permissible distances between elements, geometric intersections, logical relationships between elements, and so on. 
- Exchange of information - structured collection and reporting of information that meets the format requirements and the level of confidence per $m$ of pre-defined stages of the project.

- IFC is a universal format for standard data exchange between BIM applications.

\section{Step-by-step BIM design technology implementation plan}

\section{STEP 1. Transition from computer-aided design to BIM information design. (Laying the groundwork for BIM adoption.)}

\section{Customer's enterprise audit}

At the first stage, the audit of the enterprise:

- Conducting interviews with managers to determine the goals and objectives of implementing BIM technology (in person or remotely).

- Interviews with key professionals to understand existing design and collaboration technologies and identify their strengths and weaknesses (in person or remotely).

- Collecting information about the characteristics of the work and the maturity of BIMtechnologies in the client's company.

- Analysis: the format of interaction between departments, standards for the design of materials for project documentation, the specifics of designed objects, sources of information for the BIM model.

- Object selection for the pilot project of the most typical 's customer objects.

- Preparation of detail of description I services for the implementation of BIM technologies, including 3D, 4D, 5D, 6D and 7D.

The detailed terms of reference gives the contractor and the customer the same understanding of the scope of work that is intended for the implementation of the implementation project.

Based on the results of the audit, the terms of reference for the implementation and transition to BIM technology are developed and approved.

\section{BIM implementation schedule development}

The BIM implementation plan is developed as a result of the analysis of audit materials, and the developed and agreed technical task for implementation

To comply with the schedule, a calendar plan is drawn up for the activities necessary for the transition to BIM technology.

- for training employees, it is necessary to prepare free premises equipped with a PC with installed software, with a sufficient number of workplaces should be sufficient for training;

- the choice of the training schedule (one course of study (40 academic hours) can be carried out both within one week (training lasts a full working day), and over longer periods.

- coordination structures s meetings in developing BIM Deployment Plan.

\section{Development of the necessary infrastructure to ensure the implementation of BIM}

At this stage: 
- the structure of the team involved in the project is determined;

- the job responsibilities of each participant are formed;

- methods of communication between all project participants are being developed;

- requirements for IT infrastructure and technical support are being developed;

- requirements for templates and libraries, quality control procedures and change management methods are being developed.

\section{Formation of requirements for hardware, information and software}

The information modeling process requires the following resources:

- software integrated into a single information environment in accordance with the organization's BIM strategy;

- hardware of providing I, allowing the first to implement BIM technologies, including server hardware and jobs of designers, including deleted;

- network resources to ensure the exchange of data between workstations and the server in order to organize collective work on BIM projects in real time;

- BIM content / libraries Resources containing components (families), project templates, and family templates that are used in BIM projects and are hosted on a file server.

\section{Preparing for implementation}

The preparation includes the creation of project templates and library files for specialists of each section provided for by the TOR.

The initial data for this stage are the results of the customer's enterprise audit.

During the preparation, materials are formed for training, and further work of the customer's specialists.

The list of documents is approved at the stage of development of the terms of reference and the implementation schedule.

Preliminary list of documents under development:

- Development of BIM standards, taking into account the specifics and peculiarities of the project company, simplifying the exchange of data through standard formats (including through the IFC format) when using various programs for BIM modeling.

- Regulations for different departments, describing in detail the processes of creating, transferring and storing design / construction tasks.

- A project template that contains loaded families, preset views, filters, created specifications, sheets, etc., in order to simplify and standardize the design process.

- Family templates that contain the necessary source data and settings to create new loadable families of specific categories.

- Methodical materials

- Requirements for the 3D model

The list is modified for a specific project and tasks.

\section{STEP 2. Testing BIM technology}

\section{Pilot training}

Training is built as a continuous process that accompanies implementation and support. Various methods of employee training are used, depending on the level of knowledge and 
experience in the software. The training starts from the Basic level (2-3 days) and ends with the Specialized level (5-15 days), after each level, the acquired knowledge is checked.

The training of designers is supposed to be carried out in stages, from formed groups of specialists from various sections.

For the first part of the implementation, the training includes the following approximate steps:

1. Employee entrance testing

2. Development of individual training courses and programs based on the results of entrance testing.

3. Organization and conduct of employee training

○ Basics of work

- Creating families

- Collaboration

- Registration of working documentation

- Interchange procedures for compatible data.

- Basic model-based analysis: visualization, collision detection, 2D and 3D calculations.

○ 4D, 5D, 6D and 7D modeling technologies.

4. Testing and analysis of employee performance

At the end of the course, students undergo mandatory testing to determine the quality of assimilation of new knowledge.

\section{Development of a pilot project}

A pilot project is an independent project development by the customer based on a new technology under the guidance of the contractor's specialists.

The interaction of the customer's designers and the contractor's specialists is carried out in two formats - face-to-face and remote support.

The implementation of the project begins at the contractor's territory, where an expert oversees the project creation process.

In the future, the contractor's specialist will be involved as remote support in the design process and will help solve any problems that arise during the design using new technologies.

Contractor daily checks information model for the purpose of detecting errors and bottlenecks (compliance, multi-disciplinary inspection and so on. D.), Taking place in the project Dalen monitoring of compliance with the standard, holding discussions and consultations on the project structure and the related changes in work e software provision.

\section{Developing collaboration procedures}

- Planning a collaboration strategy

- Formation of joint work activities

- Timeline for model development, exchanges of information for submission and confirmation

- Interactive work environment

- Digital communication procedures

\section{Adjustment of regulatory documents, templates and libraries}

In the process of training and bringing a pilot project, there is a closer interaction of the contractor's experts with the customer's designers, which allows in a short time to determine the necessary degree of revision of regulatory documents, templates and libraries. 


\section{STEP 3. Scaling BIM technology to all participants in the process}

The purpose of this section is to develop interoperability, data integration and engineering calculations

- Modeling standards are being extended to new types of projects.

- Advanced engineering data management technologies are being introduced, integrated into the collective production process..

- More emphasis is placed on sharing information and metadata.

- New types of calculations and analyzes are performed, such as budgeting and planning (4D and 5D).

Technology redesign to other design groups can be carried out with the involvement of CAD specialists or with the participation of the Contractor's specialists, depending on the customer's choice.

\section{STEP 4. Operation and integration of BIM technology}

Creation of an integrated environment for all specialists, allowing to efficiently carry out projects, carry out technical calculations and manage the operation of objects.

- High level of management with emphasis on quality and ease of use.

- BIM models and standards are stable and can be used universally with high efficiency and benefits, including economic benefits.

- Collaboration moves to a higher level. Based on the model, enhanced services are offered, its availability is increased and its relevance is ensured.

- Opportunities appear for a more complete analysis of the environmental performance of the project at all stages of its life cycle from the design to the organization of work.

When implementing the implementation plan, it is necessary to schedule a series of meetings for the project teams, discussing key issues.

\section{Approximate structure of meetings when developing a BIM implementation plan}

At least four meetings are planned, depending on the results achieved

\section{Session 1 Organizational.}

1. Discussion of BIM experience in the implementation of information technology.

2. Development of BIM goals of the organization.

3. Identification of BIM applications.

4. Development of the 1st level BIM process map.

5. Identification of responsible parties for the development of process maps of the 2 nd level.

6. Scheduling of subsequent meetings

7. Any other business.

8. Coordination of tasks, time of their implementation and responsible parties.

\section{Session 2. Development of BIM processes}

1. Coordination and approval of process maps, containing boiling detailed plan information process BIM design.

2. An overview of the initial BIM goals and applications. 
3. Overview of the 1 st level process card

4. Analyze workflows from different sources to identify overlaps between different information modeling tasks

5. Review the process to identify opportunities and challenges

6. Determination of the main exchanges of information in accordance with the design processes.

7. Identify a responsible party to coordinate each exchange of information, including exchanges between the user and the author.

8. Coordination of interdisciplinary teams and the development of rules for the exchange of information between them.

9. Any other questions.

10. Coordination of tasks, timing of their implementation and responsible parties.

\section{Session 3. Development of information exchanges and identification of related infrastructure.}

1. Alignment formed BIM acknowledgment purposes and is, their Match to Ia initial targets.

2. Overview of the developed approaches and procedures exchange and information.

3. Assertion of infrastructure needs to support processes and information exchanges.

4. Any other questions.

5. Coordination of tasks, timing of their implementation and responsible parties.

\section{Session 4 Review of intermediate results.}

1. Review of the results of the development of a pilot BIM project.

2. Coordination of the developed control technologies for the implementation of the design work plan.

3. Approval of procedures for adaptation and monitoring of the project implementation plan.

4. Any other questions.

5. Coordination of tasks, time of their implementation and responsible parties.

\section{Conclusion}

Summing up, it should be noted that the presented step-by-step plan for the implementation of information modeling technology when used by a design organization can be modified and supplemented in accordance with the characteristics of the tasks and projects being performed, however, this development can significantly reduce the time for the implementation of BIM technologies.

\section{References}

1. Dmitrieva I.S., Gariaev N.A. Application of simulation modeling for the analysis of architectural and planning solutions for unique objects. In the collection: information systems, technologies and automation in construction. Electronic resource: collection of scientific papers of the Department of ISTAS NRU MGSU. Moscow, 2015.S. 56-60. 
2. Gariaev N.A., Gariaeva V.V., Rybina A.V. Development of a simulation model for the analysis of design solutions for remote construction objects from the point of view of providing building materials and structures. Scientific Review. 2015. No. 13. S. 395398.

3. Garyaev N.A. Design and analysis of information model hotel complex. MATEC Web of Conferences 2016. C. 06007.

4. Garyaev N.A. Geographically distributed learning network construction industry. Computing in Civil and Building Engineering Proceedings 2014 International Conference. 2014. C. 1578-1585.

5. Garyaev N.A., Gagarin P.V. Modern computational modeling as a stage in the development of the design process. MGSU Bulletin. 2009. No. S1. S. 186-187.

6. Garyaev N.A., Krasnoshchekova A.I., Knyazev A.A. Analysis of the risks arising from the implementation of BIM-technologies in construction organizations. BST: Construction Technique Bulletin. 2018. No. 7 (1007). S. 58-61.

7. Garyaev N.A., Rybina A.V. Simulation model of material and technical support of construction objects. System technologies. 2018. No. 1 (26). S. 142-150.

8. Garyaev P.N. Computer-aided zoning and urban planning. Computing in Civil and Building Engineering Proceedings 2014 International Conference. 2014. C. 1618-1625.

9. Garyaeva V.V. Application of BIM modeling for the organization of collective work on a construction project/ MATEC Web of Conferences 2018. C. 05025. 